\title{
Dimensioning Cellular Networks with Fixed Relay Stations
}

\author{
Josep Vidal \\ Signal Processing and Communications Group \\ Technical University of Catalonia (UPC) \\ 08034 Barcelona, Spain
}

\author{
Ninoslav Marina and Anders Høst-Madsen \\ Department of Electrical Engineering \\ University of Hawai'i at Mānoa \\ 2540 Dole Street, Honolulu, HI 96822
}

\begin{abstract}
We analyze the downlink spectral efficiency of multicellular wireless networks where in each cell in addition to the base station (BS) a certain number of relay stations (RS) are deployed. It is observed that by introducing more relay stations, the spectral efficiency in $\mathrm{b} / \mathrm{s} / \mathrm{Hz}$ per cell area increases. Hence, for a fixed spectral efficiency, the cell radius can be increased. These two aspects are important since by deployment of relay stations, the existing cellular systems could get better efficiency and the new cellular system could be designed with a smaller density of BS on the same geographical area. Deployment costs can therefore be significantly reduced depending on the relative costs between RS and BS.
\end{abstract}

\section{INTRODUCTION}

Assisting relays are a promising solution being currently considered to enhance wireless communications in terms of increased data rate and robustness against the channel impairments. It is based on the three-terminal network considered in [1][2], where it was assumed that the relay terminal operate in full-duplex mode. However, that assumption is unrealistic in practical systems, and the relay operation must be divided in two orthogonal phases: the relayreceive phase and the relay-transmit phase. The achievable rates for different transmission schemes using a single halfduplex relay are considered in [3][6].

The use of relay stations offers a great potential in wireless communications in terms of increasing the transmission rates. One example where the deployment of relay stations enhances the system performance is in the downlink transmission, i.e. transmission from the base station (access point) to the mobile terminals. In a cellular network, direct transmissions between the base station and users close to the cell boundary can be very expensive in terms of the transmission power required to ensure reliable communication at high bit rate.

In this paper we evaluate the system performance with the objective of deriving general guidelines for cell design of relay-enhanced systems. We observe that by deploying relay stations in each cell, the system performance is improved in terms of :

- Increased average spectral efficiency for a given cell radius,
- Increased cell radius for a given spectral efficiency, and hence lower density of BS deployment, and

- Enhanced peak bitrate for users close to the cell boundary.

In order to analyze the performance of the relay based system, we evaluate two fundamental measures that capture most of the benefits offered by relay-based transmission. One of them is the cellular spectral efficiency (CSE), as the average achievable rate over the cell area, and the other is the outage minimum cell-wide achievable rate (MAR), as the peak achievable rate of the $\varepsilon$-percentile worst users in the cell. The purpose of this paper is to assess that gains may be achieved when relays are deployed, and under which conditions these gains may be observed.

The organization of the paper is as follows: Section 2 describes the system model and the relaying transmission protocol. Section 3 defines a model for evaluation of the spectral efficiency. Section 4 displays the system performance with respect to position and number of relays. Section 5 outlines the procedure used to dimension the cells while Section 6 concludes the paper.

\section{SYSTEM MODEL}

We consider the half-duplex scenario shown in Error! Reference source not found., where the BS transmits the data to the mobile station (MS) either directly or via the RS in two orthogonal hops, depending on the strategy achieving largest rate. The duration of each hop is variable and optimized so as to maximize the transmission rate. All terminals may be equipped with a single or multiple antennas.

The RS are distributed uniformly in angle, all at the same distance $r_{B R}$ of the BS. The transmission is carried out in frames of length $T$ and bandwidth $\mathrm{W}$ (both parameters will be normalized to one). The relays operate in half-duplex mode with a duration $\alpha \mathrm{T}_{\text {frame }}$ and $(1-\alpha) \mathrm{T}_{\text {frame }}$ for the relay-receive and relay-transmit phase, respectively. The adjustment of the duration of the transmission phases allows enhanced transmission performance [6].

J. Vidal was supported by the European Union through the project FIREWORKS IST-4-027675, by the Spanish/Catalan Science and Technology Commissions and FEDER funds from the EC: PR2006-0456, TEC200606481/TCM, TEC2004-04526, and 2005SGR-00639.

$\mathrm{N}$. Marina has been supported by the Swiss National Science Foundation Grant PA002-117385. 


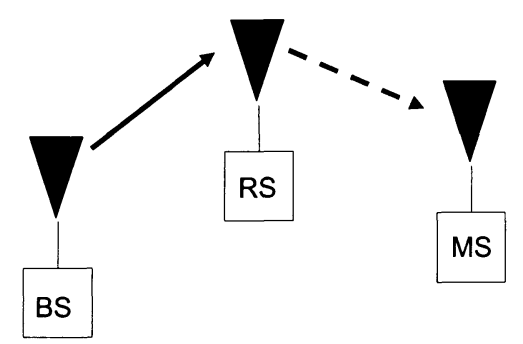

Figure 1. Each cell features several relay stations. The scheduler decides to transmit either directly from BS to MS or through two orthogonal hops.

The received signals at the mobile terminal $i$ on a single carrier is given by

$$
\mathbf{y}_{M, i}^{(1)}=d_{B M}^{-\beta_{2}} \mathbf{H}_{B M} \mathbf{x}_{B, i}+\mathbf{z}_{M, i}^{(1)}
$$

in the case where there is a direct transmission from the BS to MS. For relayed transmission, the signals at the mobile terminal $i$ and its associated $\mathrm{RS}$ are

$$
\begin{gathered}
\mathbf{y}_{R, i}=d_{B R}^{-\beta_{1}} \mathbf{H}_{B R} \mathbf{x}_{B, i}+\mathbf{z}_{R, i} \\
\mathbf{y}_{M, i}^{(2)}=d_{R M}^{-\beta_{2}} \mathbf{H}_{R M} \mathbf{x}_{R, i}+\mathbf{z}_{M, i}^{(2)} .
\end{gathered}
$$

Here $d_{B M}, d_{B R}$ and $d_{R M}$ are the distances between BS and MS, BS and RS and RS and MS, respectively. Matrices $\mathbf{H}_{\mathbf{B M}}$, $\mathbf{H}_{\mathbf{B R}}$ and $\mathbf{H}_{\mathbf{R M}}$ account for the spatial channel in each link. The dimension of each $\mathbf{H}$ matrix is the number of receive times the number of transmit antennas. It is assumed that there is always a line-of-sight (LOS) between the BS and RS and there is no line-of-sight (NLOS) between BS and MS and RS and MS. This is a realistic assumption for a scenario where all BSs and RSs are above the roof tops. Therefore, the path loss exponent in the link BS-RS is assumed to be $\beta_{1}$, while for the links BSMS and RS-MS it is assumed to be $\beta_{2}$ (see Error! Reference source not found.). $x_{B}$ and $x_{R}$ are the transmitted signals from the BS and RS, respectively, while $y_{R}$ and $y_{M}$ are the received signals at the RS and MS, respectively. The power input constraints are $E\left\{\left|x_{B}\right|^{2}\right\} \leq P_{B}$, and $E\left\{\left|x_{R}\right|^{2}\right\} \leq P_{R}$ at the BS and $\mathrm{RS}$ respectively. Terms $\mathrm{z}$ include the Gaussian noise (with spectral densities $N_{R}$ and $N_{M}$ ) and the interference.

If we assume OFDM transmissions where each carrier suffers independent fading, and coding is done across carriers it is reasonable to assume capacity expressions on each link given by the ergodic capacities, $R_{B M}, R_{B R}$ and $R_{R M}$ :

$$
R=E\left\{\log _{2}\left(\mathbf{I}_{N}+\frac{P}{W N+I} \mathbf{H H}^{H}\right)\right\} \mathrm{bps} / \mathrm{Hz} \text {. }
$$

Exact expressions for the general correlated MIMO ergodic capacity are given in [7]. With the transmission strategy proposed, the achievable rate for user $i$ is

$$
\begin{aligned}
R_{i} & \leq \max \left\{R_{B M, i}, \max _{\alpha} \min \left\{\alpha R_{B R, i},(1-\alpha) R_{R M, i}\right\}\right\} \\
& =\max \left\{R_{B M, i}, \frac{R_{B R, i} R_{R M, i}}{R_{B R, i}+R_{R M, i}}\right\},
\end{aligned}
$$

the BS to the RS to which user $i$ is associated. Although multiplexing gain cannot be larger than that obtained for nonrelaying transmissions [4], significant gains in SNR are possible as a result of the pathloss split between the BS and the MS.

\section{MODEL For THE SPECTRAL EFFICIENCY}

To measure the average system performance we will consider that:

1. Users are deployed uniformly on the cell and have the same traffic requirements

2. Users are served by one relay at most, and each user selects the best transmission strategy, either through relaying or direct transmission

3. No user delay constraints

4. Time division multiple access.

The average spectral efficiency, CSE, is a function of the scheduling strategy selected. We might assume that the service time allocated to every user is a function of the rate achievable in equation (1), $T_{i} \propto R_{i}^{\gamma}$. The CSE obtained as an average of the achievable rates on every position in the cell $A$ weighted by the relative transmission times is therefore given as

$$
\mathrm{CSE}=\frac{\sum_{i \in A} R_{i}^{\gamma+1}}{\sum_{i \in A} R_{i}^{\gamma}} .
$$

Note that this expression encompasses different scheduling strategies. When $\gamma=0$, all users in the cell are given the same amount of time resources, so it turns to a round-robin strategy. When $\gamma>0$, the resource allocation is balanced towards the users with the best conditions.

The CSE provides an orientation of how efficient is the system and how much traffic it can deliver. Since it is an average value, it does not account for the disparity of achievable rates experienced by different users. On the other hand, the MAR assesses the benefit obtained by the worst users in terms of peak transmission rate. It is more related to the service quality that a user experiences when downloading bursty services.

Both CSE and MAR are evaluated as a function of the number of relays, the position of the relays with respect to the cell center.

\section{POSITION AND NUMBER OF RELAYS}

After the text edit has been completed, the paper is ready for the template. Duplicate the template file by using the Save As command, and use the naming convention prescribed by your conference for the name of your paper. In this newly created file, highlight all of the contents and import your prepared text file. You are now ready to style your paper; use the scroll down window on the left of the MS Word Formatting toolbar.

where $R_{B R, i}$ is the achievable rate in the transmissions from 
Based on the ergodic capacity expressions, it is relevant to determine the impact of the relay position in the system spectral efficiency in the downlink. To that end, we are using the system parameters considered displayed in Error! Reference source not found..

The results are shown in Figure 2 for a cell of $1000 \mathrm{~m}$ radius. In all cases, the CSE and MAR are compared with those obtained in direct transmissions from the source to destination (assuming NLOS situation). It can be observed that there is an optimum distance for the relay position. This is so since a relay too close to the base station will not illuminate far users, namely those having a greater need of enhanced capacity.

On the other hand, if the relay is very far from the base station, the low quality of the BS-RS link limits the capacity. Another relevant aspect to be considered is the number of RS. If the number of relays is low, the area in the cell illuminated by those relays is reduced. When increasing the number of relays, the area covered per relay reduces and the capacity tends to saturate.

TABLE I. SIMUlation PARAMETERS FOR THE DOWNLINK

\begin{tabular}{|c|c|}
\hline Parameter & \\
\hline Cellular layout & Omni-directional single cell \\
\hline Cell radius $R$ & 1,5 to $4 \mathrm{~km}$ \\
\hline Propagation exponent & $\beta_{1}=2,6$ for BS-RS \\
$\beta_{2}=3,5$ for BS-MS and RS-MS \\
\hline Carrier frequency & $3,55 \mathrm{GHz}$ \\
\hline Bandwidth & $20 \mathrm{MHz}$ \\
\hline BS power & $40 \mathrm{dBm}$ \\
\hline RS power & $36 \mathrm{dBm}$ \\
\hline BS antenna gain plus cable loss & $10,6 \mathrm{dBi}$ \\
\hline RS antenna gains plus cable loss & $5 \mathrm{dBi}$ \\
\hline User antenna gain & $-1 \mathrm{dBi}$ \\
\hline Noise spectral density & $-174 \mathrm{dBm} / \mathrm{Hz}$ \\
\hline Noise figure at MS and RS & $7 \mathrm{~dB}$ \\
\hline Body loss & $5 \mathrm{~dB}$ \\
\hline Antennas at (BS $\times$ RS $\times$ MS) & Single $1 \times 1 \times 1, \mathrm{MIMO} 2 \times 2 \times 1$ \\
\hline DL to UL frame balance & $26: 21$ \\
\hline MAC overhead & $39,7 \%$ direct transmission \\
\hline
\end{tabular}

From the plots above we conclude that the CSE is strongly related to the position of the relays, although it is not very sensitive to the exact optimal position. On its turn, the MAR is quite sensitive to the position of the relays. When a large number of relays are used, the optimum position for MAR and CSE tend to coincide. It may also be observed that both the CSE and MAR increase with the number of relays deployed and tends to saturate. The area illuminated by the relays increases with the number of RS. When both the BS and the RS are equipped with two antennas, gains are observed in those cases where the BS-RS link is limiting. This is the situation for MS situated close to the RS, when the RS is far from the BS (note that in all cases the optimum position of the $\mathrm{RS}$ is far from the BS).

\section{DIMENSIONING THE CELl RADIUS}

Relaying can provide gains as well in terms enlargement of the cell radius for a given service. The two criteria associated to service that have been adopted here are:

1. Set a cell radius $R_{C S E}$ so that the CSE that the system can cover matches the offered traffic in the cell, and

2. Set a cell radius $R_{\text {MAR }}$ so that the MAR is over a certain value.

When both CSE and MAR constraints are specified for dimensioning the cell radius $R$ it is important to have in mind that both might not be achieved simultaneously for any $R$. Let us evaluate the options in this situation.

Let us assume that $R_{C S E}$ is the radius that fits a certain cellular CSE o, and that $R_{M A R}$ is its counterpart for an objective $\mathrm{MAR}_{\mathrm{o}}$. We might have the following situations:

- $R_{M A R}<R_{C S E}$ (see Figure 3, top). As the MAR is a decreasing function of the cell radius, if we select $R=R_{C S E}$ the peak rate will be lower than the value required. On the other hand, if $R=R_{M A R}$ the cell spectral efficiency will be larger than the traffic generated by users, and hence the deployment of BS will be unnecessarily dense, resulting in uneconomical investment for the operator.

- $R_{C S E}<R_{\text {MAR }}$ (see Figure 3, bottom). As the CSE is a decreasing function of the cell radius, if we select $R=R_{M A R}$ the cell may not be able to accommodate all the traffic generated by the users, and a lack of system quality may be perceived in the unavailability of resources when needed. On the other hand, if $R=R_{C S E}$ both the peak rate and the CSE will fit the requirements.

Therefore it is necessary that both $R_{C S E}, R_{M A R}$ be as large as possible and the condition $R_{C S E} \leq R_{M A R}$ is met. The actual counterpart of Figure 3 using simulation parameters from Error! Reference source not found., SNR loss due to practical coding of $4 \mathrm{~dB}$, and the MAC overhead of 802.16 , are shown in Figure 4 and Figure 5 . The value for offered traffic is taken to be $10 \mathrm{Mbps} / \mathrm{Km}^{2}$, from an urban scenario [8]. As compared to direct transmission, it is apparent that the cell radius may be significantly enlarged provided that the number of relays is large enough: for 12 relays, the density in the number of BS may be reduced in a factor close to 2 .

Moreover, for a fixed cell radius, the gains in CSE are also important. On its turn, the MAR is significantly enhanced when the number of relays is large enough, helping to meet the requirements in terms of $R_{C S E}, R_{M A R}$ expressed before. As the CSE and the MAR are usually given in terms of bps, the bandwidth allocated to the system also plays a key role in the determination of the cell radius. A significant enlargement has also been observed (although not displayed) when the bandwidth is increased to $100 \mathrm{MHz}$. 

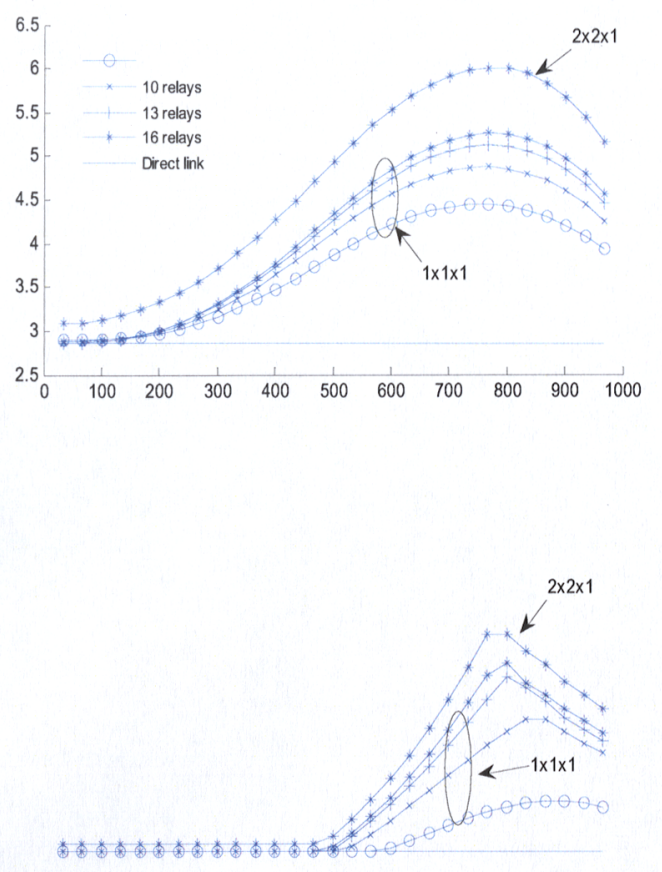

Figure 2. Spectral efficiency (top) and outage minimum achievable rate (bottom) as a function of relays position for the cell radius of $1000 \mathrm{~m}$.
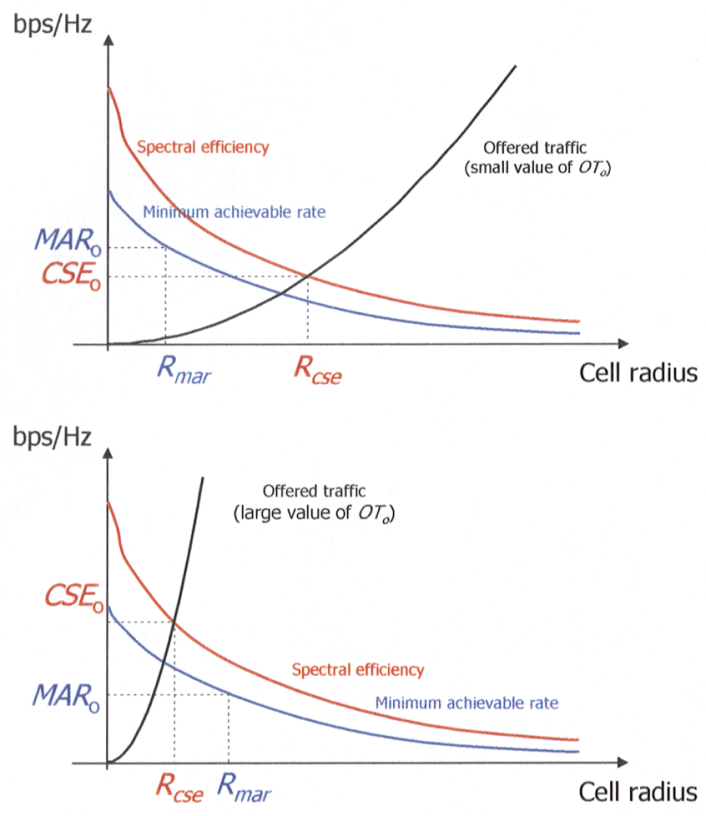

Figure 3. Cell radius selection for low offered traffic (top) and large offered traffic (bottom)
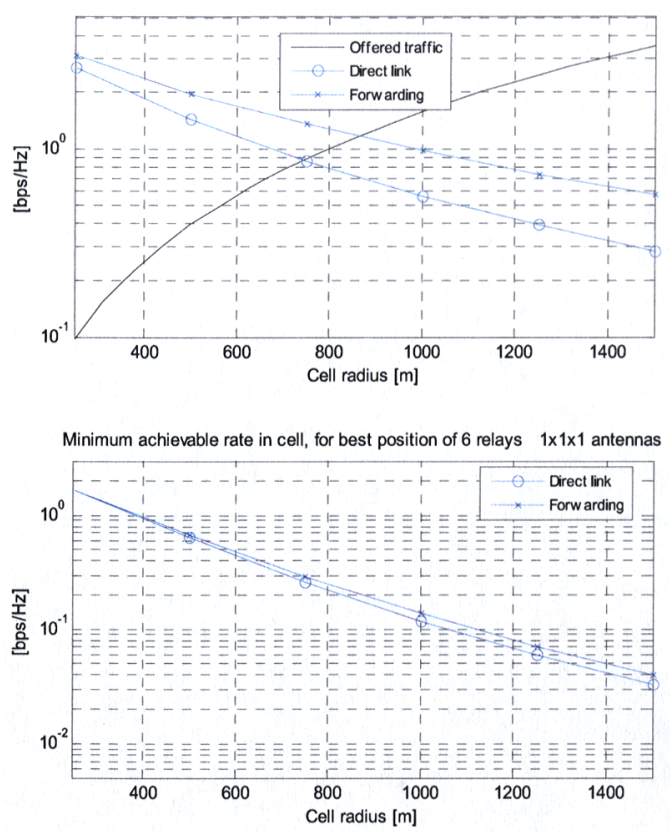

Figure 4. Cellular spectral efficiency and offered traffic (top) and minimum achievable rate (bottom) as a function of the cell radius for 6 relays.
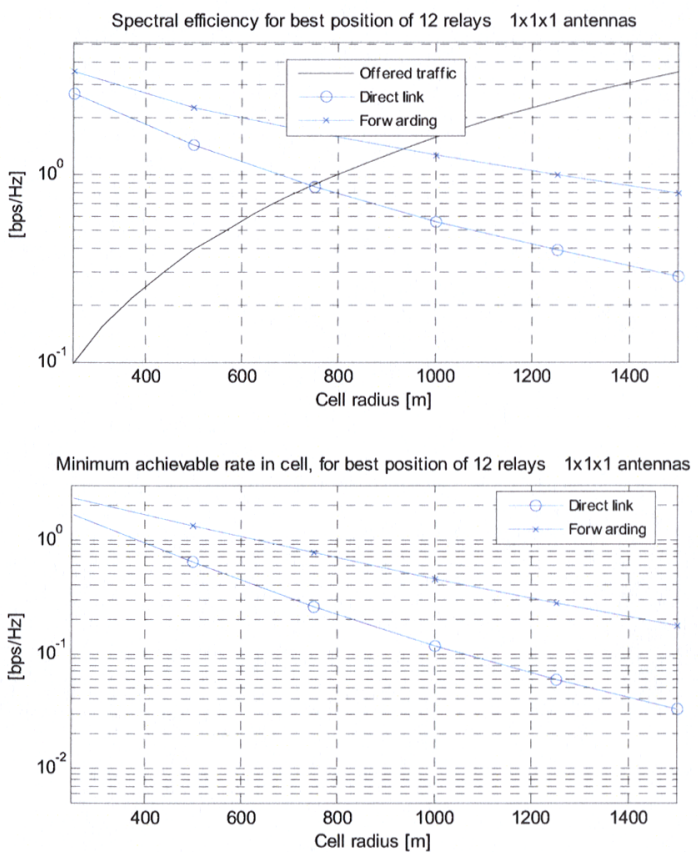

Figure 5. Cellular spectral efficiency and offered traffic (top) and minimum achievable rate (bottom) as a function of the cell radius for 12 relays. 


\section{CONCLUSIONS}

The problem of cell dimensioning for relay-assisted networks is tackled. An approach based on system level simulations has been taken to evaluate the impact of the cell radius and the number of relays in system performance. Results show that, for a fixed cell radius, significant gains can be obtained: it is possible to double the CSE as compared to direct BS-MS transmissions by using a single antenna at the BS, RS and MS. On the other hand, by fixing the offered traffic per area, the cell radius can be enlarged thus reducing the density of deployment of BS. These gains are obtained at the expenses of a deployment of RS which may be assumed centrally controlled simple terminals without higher layer capabilities. The results obtained in this paper shed some light on the way of the deployment of BSs and RSs for a given system performance.

\section{REFERENCES}

[1] E. C. van der Meulen, "Three-terminal communication channels," Adv. Appl. Prob., vol 3, pp. 120-154, 1971

[2] T. M. Cover and A. A. El Gamal, "Capacity theorems for the relay channel," IEEE Trans. on Information Theory, vol 25, no 5 pp 474-584, Sept. 1979
[3] A. Høst-Madsen and J.Zhang, "Capacity bounds and power allocation for wireless relay channels," IEEE Trans. Information Theory, vol. 51, no. 6 , June 2005

[4] A. Høst-Madsen and A. Nosratinia, "The multiplexing gain of wireless networks," Proc. of ISIT, 2005.

[5] T. M. Cover and J. Thomas, Information Theory. New York, Wiley, 1991

[6] Y. Liang and V. Veeravalli, "Gaussian Orthogonal Relay Channels: Optimal Resource Allocation and Capacity," IEEE Trans Inf Theory, vol. 51, no. 9, Sept 2005

[7] M. Kießling "Unifying Analysis of Ergodic MIMO Capacity in Correlated Rayleigh Fading Environments," European Transactions on Telecommunications, vol. 16, issue 1, 2005, pp. 17-35

[8] W. Mohr, "Data Rates Estimates and Range Calculations for new Elements of Systems Beyond IMT-2000," Presented at WWRF WG4 meeting, June 25th and 26th 2002, London, at www.wireless-worldresearch.org

[9] WINNER Project - Deliverable D3.5.1 - "Relaying concepts and supporting actions in the context of CGS," online https://www.istwinner.org/deliverables.html.

[10] H. Bölcskei, D. Gesbert, C. B. Papadias and A.-J. van der Veen, SpaceTime Wireless Systems - From Array Processing to MIMO Communications. Cambridge, Cambridge University Press, 2006. 